\title{
Thermochemistry of Nanodiamond Terminated by Oxygen Containing Functional Groups
}

\author{
Gustavo C. C. Costa ${ }^{1,2}$, Olga Shenderova ${ }^{3}$, Vadym Mochalin $^{4}$, Yury Gogotsi ${ }^{4}$ and Alexandra \\ Navrotsky ${ }^{1 *}$
}

${ }^{1}$ Peter A. Rock Thermochemistry Laboratory and NEAT ORU, University of California Davis, Davis, California, 95616, USA

${ }^{2}$ Current Address: NASA Glenn Research Center, 21000 Brookpark Road, MS 106-1, Cleveland, $\mathrm{OH}, 44135$, USA

${ }^{3}$ Nanodiamond Laboratory, International Technology Center, 8100 Brownleigh Dr., S. 120, Raleigh, North Carolina, 27617, USA

${ }^{4}$ Department of Materials Science and Engineering, and A. J. Drexel Nanomaterials Institute, Drexel University, 3141 Chestnut Street, Philadelphia, PA, 19104, USA

*Corresponding Author. Tel: 530-752-3292. E-mail: anavrotsky@ucdavis.edu (Alexandra Navrotsky) 


\begin{abstract}
The standard enthalpies of formation at $25{ }^{\circ} \mathrm{C}$ of nanodiamonds terminated by oxygen containing functional groups have been investigated by high-temperature oxidation calorimetry. Depending on the amount of oxygen containing functional groups, the nanodiamonds (plus oxygen and hydrogen as represented in the surface functional groups) can be up to $52 \mathrm{~kJ} \mathrm{~mol}^{-1}$ more stable in enthalpy than graphite, which means that less heat is evolved during oxidation of nanodiamonds terminated by oxygen containing functional groups, since their surface carbon is already partially oxidized. The stability of the nanodiamonds terminated by oxygen containing functional groups increases (enthalpy of formation becomes more negative) with increasing surface area within the studied range, reflecting the dominant effect of higher content of surface functional groups over the destabilizing effect of higher surface-to-volume ratio typical for nanoparticles.
\end{abstract}

\title{
1. Introduction
}

Diamond nanoparticles (nanodiamonds) find a wide range of applications in science and technology due to their small $(\sim 5 \mathrm{~nm})$ particle size and unique combination of properties $[1,2]$. They are commonly synthesized by detonation using explosives and by irradiation of carbon precursors using high energy beams ( $\mathrm{MeV}$ electrons) or a laser [1]. They can also be produced by crushing (milling) larger diamond particles. The properties of nanodiamonds are influenced by the synthesis method (e.g., laser synthesis vs. detonation or high-pressure high-temperature synthesis), and for many applications post-synthesis treatment is required to modify the surface chemistry of the particles, to control agglomeration, or to remove impurities, e.g. metals and their oxides [1]. Besides being produced on Earth, nanodiamonds have been detected in the Earth's mantle underneath Hawaii [3], in petroleum [4], in the interstellar medium and in primitive meteorites [5, 6]. It is also possible that nanodiamonds can be formed under hydrothermal conditions in serpentinites [7, 8]. Since $\mathrm{sp}^{3}$ carbon must form four bonds, the surface of nanodiamond is almost always terminated with functional groups or hydrogen: e.g. interstellar nanodiamonds are terminated mostly with hydrogen and nanodiamonds synthesized by detonation methods are terminated both by hydrogen and oxygen containing functional groups [1]. These functional groups are commonly described in organic chemistry as specific groups of atoms within molecules that have predictable characteristics and are responsible for 
chemical reactivity. In this work the main functional group chemically bonded to the nanodiamond surface is carboxyl $(-\mathrm{COOH})$ (carbon double bonded to oxygen and single bonded to a hydroxyl group $(\mathrm{OH})$ ). If these groups are removed from the nanodiamonds, their surfaces always undergo reconstruction to graphitic layers [1]. Thus the functional groups terminating nanodiamond particle surfaces appear to be an intrinsic part of the nanodiamond structure and are expected to strongly influence their thermodynamic stability compared to bulk diamond.

There are extensive studies on the synthesis, structure and properties of nanodiamonds including atomistic computational studies of their thermodynamic stability [1, 9, 10]. Small diamond nanoparticles have been predicted to have a higher thermodynamic stability than graphite $[9,10]$. However, no directly measured values of energetics of nanodiamonds obtained by standard experimental techniques have been reported. In this work, we measure enthalpies of formation of nanodiamonds terminated by hydrogen and oxygen containing functional groups at $25{ }^{\circ} \mathrm{C}$ using high temperature oxidation calorimetry. We show that the energetic stability of nanodiamonds increases as the specific surface area and content of carboxyl groups increases. At first glance these energetic trends present a thermodynamic anomaly since stability is expected to decrease with increasing the specific surface area due to excess surface energy. However, surface area and the amount of surface functional groups are correlated and the stabilizing effect of the latter, i.e. a less exothermic heat of oxidation due to the presence of oxygen containing functional groups on its surface, more than offsets the destabilizing effect due to the increased specific surface area.

\section{Experimental Methods}

\subsection{Materials}

Two types of nanodiamond (ND) particles were used in this study, NDs produced by detonation of carbon-containing explosives (so called detonation NDs or DNDs) and particles synthesized by a static high pressure high temperature (HPHT) process. DND powder was purchased from Real-Dzerzinsk, Russia. It had been purified from soot by the vendor using singlet oxygen (dioxygen) in $\mathrm{NaOH}$ followed by treatment in $\mathrm{HNO}_{3}$, resulting in oxidized DND terminated mainly by carboxyl groups. While sizes of primary particles of DND are 4-5 nm, the average aggregate size of the as-purchased polydispersed material in water was $150 \mathrm{~nm}$, as measured with dynamic light scattering. These aggregates are tight and unbreakable by 
sonication. Samples with aggregate sizes of $40 \mathrm{~nm}(\# 1)$ and $180 \mathrm{~nm}(\# 2)$ were produced using fractionation by centrifugation of DND slurry in deionized water. HPHT nanodiamond powder samples with aggregate sizes of $35 \mathrm{~nm} \mathrm{( \# 3)} \mathrm{and} 100 \mathrm{~nm}$ (\#4) were purchased from Microdiamant, Switzerland. These HPHT NDs are monolithic particles.

\subsection{Characterization}

The nanodiamond powder samples were probed by infrared spectroscopy using a Bruker Equinox 55 FTIR spectrometer to detect the presence of $\mathrm{H}_{2} \mathrm{O}$, functional groups and adsorbed molecules on their surfaces. Spectra of $\mathrm{KBr}$ pellets containing these powdered samples were recorded from 400 to $4000 \mathrm{~cm}^{-1}$. Blank subtractions were performed using pure $\mathrm{KBr}$.

Powder X-ray diffraction (XRD) patterns of the nanodiamond samples were recorded using a Bruker-AXS D8 Advance diffractometer (Bruker-AXS Inc.) operated at an accelerating voltage of $40 \mathrm{kV}$ and an emission current of $40 \mathrm{~mA}$ with $\mathrm{CuK} \alpha$ radiation $(\lambda=0.15406 \mathrm{~nm})$. Data were acquired from 15 to $95^{\circ} 2 \theta$ with a step size of $0.03^{\circ}$ and a collection time of $0.5 \mathrm{~s} \mathrm{step}^{-1}$. The sample was rotated at $15 \mathrm{rpm}$. Crystalline phases were identified using Jade 6.1 software (Materials Data Inc,) equipped with both the International Center of Crystallographic Data (ICCD) Powder Diffraction File (PDF) and the International Center of Structure Data (ICSD) database. Crystallite size was calculated from diffraction peak broadening using a whole profile fitting procedure (Williamson-Hall (W-H) analysis) [11] as implemented in the Jade 6.1.

Specific surface areas (SSA) were determined by $\mathrm{N}_{2}$ adsorption using the Brunauer-EmmettTeller (BET) isotherm at $-196^{\circ} \mathrm{C}$. Ten-point adsorption isotherms of nitrogen were collected in a relative pressure range of $\mathrm{p} / \mathrm{p}^{0}=0.05-0.3\left(\mathrm{p}^{0}=\right.$ saturation pressure, 760 torr for $\left.\mathrm{N}_{2}\right)$ using a Micromeritics ASAP 2020 surface area and porosity analyzer. Prior to analysis, the samples were degassed under vacuum at $200{ }^{\circ} \mathrm{C}$ for $2 \mathrm{~h}$. The uncertainties in the BET surface area measurements were propagated from the fitting of the straight line $1 /\left[\mathrm{Q}\left(\mathrm{p}^{0} / \mathrm{p}-1\right)\right](\mathrm{Q}=$ quantity adsorbed, mmol $\mathrm{g}^{-1}$ ) vs $\mathrm{p} / \mathrm{p}^{0}$ by the Micromeritics software and were calculated using the equation described in the Micromeritics ASAP2020 operator's manual.

The content of carboxyl groups on the surface of the carboxylated nanodiamond samples was quantified by a modified Boehm titration method [12]. The principle of this method is similar to back titration where the amount of carboxylic sites is determined by reacting them with a known excess of sodium bicarbonate solution. The remaining sodium bicarbonate is then titrated with hydrochloric acid to calculate how much sodium bicarbonate reacted with carboxylic acid sites. In the modified Boehm titration method, $0.2-0.4 \mathrm{~g}$ of the carboxylated 
nanodiamond samples were dispersed in $25 \mathrm{~mL}$ of the $0.0487 \pm 0.0003 \mathrm{~mol} \mathrm{~L}^{-1} \mathrm{NaHCO}_{3}$ standardized solution and stirred under nitrogen overnight. The dispersion was titrated potentiometrically with $0.100 \pm 0.001 \mathrm{~mol} \mathrm{~L}^{-1} \mathrm{HCl}$ standard solution using a $\mathrm{pH}$ electrode with a T50 Mettler Toledo titrator (Mettler Toledo International Inc., Leicester, UK).

\subsection{High Temperature Oxidation Calorimetry}

High-temperature oxidation calorimetry was performed using an AlexSys Setaram isoperibol Tian-Calvet twin microcalorimeter to determine the enthalpy of oxidation of the nanodiamond powder samples. $\sim 1 \mathrm{mg}$ pellets were loosely pressed, weighted, and dropped from room temperature $\left(25^{\circ} \mathrm{C}\right)$ into a silica glass crucible with a small piece of silica wool at its bottom in the calorimeter at $800{ }^{\circ} \mathrm{C}$. In these experiments the samples were rapidly oxidized to $\mathrm{CO}_{2}$ gas at calorimeter temperature by the reaction: $\mathrm{C}_{\left(\mathrm{s}, 25{ }^{\circ} \mathrm{C}\right)}+\mathrm{O}_{2\left(\mathrm{~g}, 800{ }^{\circ} \mathrm{C}\right)} \rightarrow \mathrm{CO}_{2}\left(\mathrm{~g}, 800{ }^{\circ} \mathrm{C}\right)$. Oxygen was flushed through the area close to the silica wool at $40 \mathrm{~mL} \mathrm{~min}^{-1}$ and through the calorimeter assembly at $50 \mathrm{~mL} \mathrm{~min}{ }^{-1}$ to ensure full oxidation of samples to $\mathrm{CO}_{2}$. Measurements were repeated 5 to 9 times to achieve statistically reliable data. The calorimeter was calibrated against the heat content of platinum. The procedure was similar to that used earlier for carbon nanotubes and onion-like carbons $[13,14]$.

\section{Results}

All nanodiamond samples used for calorimetry were confirmed to be XRD pure phases (the only crystalline phase detected was cubic diamond), as shown in Fig. 1. The nanocrystalline nature of the samples results in diffraction peak broadening. Powder XRD was used for measuring the crystallite size, and nitrogen adsorption was used for measuring the particle or agglomerate size (see Table 1). The crystallite sizes of the samples calculated from whole pattern fitting (XRD) are larger than those calculated from nitrogen adsorption, which could be related to the assumptions of the model (spherical particles when deriving the size from BET data for ND) or could occur because the precision and sensitivity of BET analysis are higher when the particle size becomes smaller (SSA becomes larger) in contrast to XRD, where the precision is higher when the size is larger. Thus, SSA derived particle size distribution is dominated by the smaller particles whereas the XRD derived one is dominated by the larger particles. As described earlier $[15,25]$ for nanocrystalline powder samples of metal oxides, the particle sizes measured by XRD and nitrogen adsorption are consistent with those obtained by electron microscopy, so 
the employment of this third technique would not change the course of the experimental findings.

The presence of adsorbed water, hydroxyl and carboxylic groups was detected by FTIR analysis (Fig. 2) in the carboxylated nanodiamond samples [16, 17]. The Fourier transform infrared (FTIR) spectrum of the carboxylated nanodiamond samples \#1, \#2 and \#3 exhibited bands at $\sim 3420 \mathrm{~cm}^{-1}, 1756 \mathrm{~cm}^{-1}$ and $1625 \mathrm{~cm}^{-1}$. The two bands at $\sim 3420 \mathrm{~cm}^{-1}$ and $1625 \mathrm{~cm}^{-1}$ correspond to stretching mode $\left(\mathrm{v}_{\mathrm{OH}}\right)$ and bending mode $\left(\delta_{\mathrm{OH}}\right)$ of the hydroxyl groups terminating the ND surface and/or originating from adsorbed water [18]. The band at $1756 \mathrm{~cm}^{-1}$ is attributed to $\mathrm{C}=\mathrm{O}$ stretching mode $\left(\mathrm{v}_{\mathrm{C}=\mathrm{O}}\right)$ of carbonyls or carboxyl groups on the nanodiamond surfaces. Note that the relative intensity of all bands in Fig. 2 increases with increasing amount of oxygen in the samples as shown in Fig. 4. The amount of oxygen was calculated from the amount of carboxyl groups measured by the Boehm titration. Thus, the intensity of the transmittance bands is in fact proportional to the amount of carboxyl groups and adsorbed water. Adsorbed water is retained in the samples due to hydrogen bonding and Van der Waals and London interactions with the carboxyl groups. Sample \#4 exhibited only two bands at $\sim 3420 \mathrm{~cm}^{-1}$ and $\sim 1600 \mathrm{~cm}^{-1}$ corresponding to the stretching mode $\left(\mathrm{v}_{\mathrm{OH}}\right)$ and bending mode $\left(\delta_{\mathrm{OH}}\right)$ of the surface hydroxyl groups or adsorbed water. This sample has the lowest amount of carboxyl groups (Table 2) which explains the absence of the $\mathrm{v}_{\mathrm{C}=\mathrm{O}}$ vibrations at $\sim 1756 \mathrm{~cm}^{-1}$.

The enthalpies of oxidation of the carboxylated nanodiamond samples and other allotropes and their enthalpies relative to graphite are presented in Table 3 and Fig. 3. The thermodynamic cycle in Table 4 is used to calculate the enthalpies of oxidation of the nanodiamond samples at $25^{\circ} \mathrm{C}\left(\Delta \mathrm{H}_{\mathrm{ox}}\right.$, sample, $\left.25^{\circ} \mathrm{C}\right)$ since the oxidation was done at $800{ }^{\circ} \mathrm{C}$. The variables $a, b, c$ and $d$ of the thermodynamic cycle are given in Table 5. A brownish residue was formed in the calorimeter after the oxidation of the nanodiamond samples and is assumed to be mainly composed of hematite $\left(\mathrm{Fe}_{2} \mathrm{O}_{3}\right)$ [1]. A calculation, based on the amount of water and iron oxide measured by gravimetric analysis, shows that the contribution of enthalpy effects due to any iron oxidation and water vaporization in samples is below the calorimetric uncertainties $\left(0.5-2.4 \mathrm{~kJ} \mathrm{~mol}^{-1}\right)$.

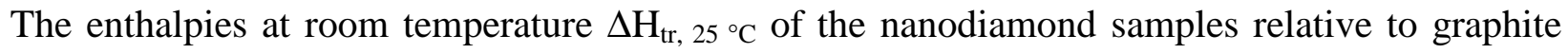
(plus $\mathrm{O}_{2}$ and $\mathrm{H}_{2}$ in concentrations corresponding to $\mathrm{O}$ and $\mathrm{H}$ content in the surface functional

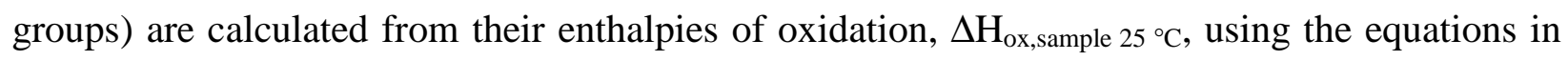
Table 4 , and from the enthalpy of oxidation of graphite $\Delta \mathrm{H}_{\mathrm{ox}}$ according to the thermodynamic 
cycle in Table 6. The enthalpies of oxidation are presented in Table 3 and in Fig. 3. The enthalpies of oxidation of the nanodiamonds terminated by carboxyl groups become more endothermic (implying stabilization) as the surface area and the content of these functional groups increases. As shown in Fig. 4, there is a linear correlation between the amount of oxygen from the carboxyl groups on the nanodiamond surface and their enthalpy of formation at room temperature up to $\sim 0.006 \mathrm{~mol}$. After this point, the enthalpy of oxidation is the same within experimental error for the sample \#1 with 0.015 moles of oxygen. Note that the total amount of oxygen is calculated from the total amount of carboxyl groups determined by titration. The carboxyl groups in the interface region of the particles are not always exposed in solution to the titrant implying a possible underestimation of the total amount of carboxyl groups in samples with large aggregates or with more interfaces. This means that the total amount of carboxyl groups in the sample \#2 with larger aggregates $(180 \mathrm{~nm})$ exposed in solution to the titrant may be less than those in the sample \#1 with smaller aggregates $(40 \mathrm{~nm})$. This correlation of the enthalpy of oxidation with the amount of oxygen from the carboxyl groups on the nanodiamond surface is similar to that observed for carboxylated herringbone multiwalled carbon nanotubes [20]. Among the samples studied here, carboxylated nanodiamonds are $14-52 \mathrm{~kJ} \mathrm{~mol}^{-1}$ more stable than graphite (plus $\mathrm{H}_{2}$ and $\mathrm{O}_{2}$ ).

\section{Discussion}

The trend toward less exothermic enthalpy of oxidation with increasing amount of carboxyl functional groups implies energetic stabilizing effects of these groups on the surface of nanodiamond particles. It has been previously observed for metal oxides that water and other surface groups such as hydroxyl and carbonate stabilize their surfaces [25]. The energetic contribution of these groups to the nanoparticle surface energy has been used as a correction factor for calculation of the energy of the bare nanoparticle surfaces based on calorimetric measurements [25]. In the case of nanodiamond, surface functional groups are an integral part of the structure, since the dangling bonds on the surface of the $\mathrm{sp}^{3}$ carbon clusters must be terminated or transformed into $\mathrm{sp}^{2}$ carbon [1]. Therefore, the nanodiamond surface functional groups are not corrected for here by subtracting an enthalpy associated with their formation. Rather, the entire nanodiamond particle, with possible surface reconstruction and functional groups, is considered to be the system of interest. The carboxyl functional groups bonded to 
nanodiamond surfaces stabilize the whole structure, so the enthalpies of oxidation of the nanodiamond samples become less negative as the content of these groups increases (Fig. 4). In a simplistic interpretation, nanodiamonds with oxygen containing functional groups can be considered already partially oxidized, and therefore release less heat during subsequent complete oxidation to $\mathrm{CO}_{2}$ and $\mathrm{H}_{2} \mathrm{O}$. Thus, their enthalpy relative to graphite plus $\mathrm{O}_{2}$ and $\mathrm{H}_{2}$ in the amounts corresponding to $\mathrm{O}$ and $\mathrm{H}$ present in the functional groups become more negative (increasing stability) as the content of functional groups increases. Similar stabilization by functional groups was found for the carbon onion sample UD50 $1300{ }^{\circ} \mathrm{C}$ (Table 3 and Fig. 3) which is about $13 \mathrm{~kJ} \mathrm{~mol}^{-1}$ less exothermic than the other carbon onion samples with higher specific surface area [14]. Our results corroborate the calorimetric observations by Cherkasov et al. [20], who found a linear correlation between standard enthalpy of formation of carboxylated herringbone multiwalled carbon nanotubes (MWCNTs) and the oxygen content determined by thermogravimetric analysis. They also found that enthalpies of formation at $25{ }^{\circ} \mathrm{C}$ of the MWCNTs are negative for the samples with oxygen containing functional groups.

The contribution of oxidized surface carbon atoms in the enthalpy of oxidation of nanodiamond can be estimated by subtracting these oxidized surface carbon atoms from the total number of carbon atoms in a $5 \mathrm{~nm}$ spherical diamond particle consisting of 11686 carbon atoms. 1560 of these atoms are on the surface and have less than 4 bonds to other carbon atoms. Only those undercoordinated carbon atoms exposed on the nanodiamond surfaces can bind to oxygen and form oxygen containing functional groups. For a simple estimate of energetics, we assume that all of these 1560 carbon atoms behave as though they were fully oxidized and do not contribute to the enthalpy of oxidation when dropped into the calorimeter. Then the remaining number of oxidizable carbon atoms in a $5 \mathrm{~nm}$ nanodiamond particle is $11686-1560=10126$ or $86.65 \%$ of the total carbon, and, accordingly, the enthalpy of oxidation of one mole of oxidizable carbon in nanodiamond sample \#1, for instance, would be $(-344.08 \pm 2.03) / 0.8665=-$ $397.09 \pm 2.34 \mathrm{~kJ} / \mathrm{mol}$. This is the same, within experimental error, as the enthalpy of oxidation of bulk diamond, $-395.42 \pm 0.50 \mathrm{~kJ} / \mathrm{mol}$. Therefore, this simple estimate confirms that the presence of carboxyl groups on the surface can account for the apparent stabilization of nanodiamond compared to bulk diamond. 


\section{Conclusions and Implications}

Our results confirm that nanodiamonds terminated by oxygen containing functional groups are much more stable in enthalpy (produce less heat during oxidation) than bulk graphite and diamond allotropes. Oxygen containing groups are already partially oxidized carbon, which produces less heat upon oxidation to $\mathrm{CO}_{2}$ compared to unoxidized carbon, i.e., to a hypothetical nanodiamond particle of the same size with a bare $\mathrm{sp}^{3}$ carbon surface. These results suggest that the surface functional groups on nanodiamond are an essential part of its structure and thus play an important and likely essential thermodynamic role in stabilizing nanodiamond and enabling its formation and persistence. Without taking the surface functional groups into consideration, it would be difficult to explain the experimental thermodynamic properties of nanodiamonds, as well as other nanoparticles, whose surface-to-volume ratio is high and whose structure is such that the surface atoms must be terminated by functional groups. Equally importantly, this calculation emphasizes the care with which composition must be defined for materials on the nanoscale. A mole of nanodiamond does not mean simply one mole of carbon (as would be assumed for bulk diamond) but rather one mole of material, consisting of (1-x) moles of carbon (which in turn may contain atoms with several types of bonding) and x moles of heteroatoms or functional groups in the structure. To interpret thermodynamic and other properties of such composite systems in detail, both composition and bonding state of the heteroatoms must be characterized accurately.

Such surface functional groups may in fact be necessary for nanodiamond to form and persist. They may retard coarsening, crystal growth, and further oxidation both in a thermodynamic sense by the added energetic stability they provide and in a kinetic sense by protecting the surface. The added thermodynamic stability of such functionalized nanodiamond relative to graphite may explain its occurrence under synthetic or natural conditions where bulk diamond would not be stable.

\section{Acknowledgments}

The calorimetric studies at Davis were supported by the Deep Carbon Observatory funded by the Alfred P. Sloan Foundation. Carbon phase synthesis and characterization at Drexel University was supported by the Fluid Interface Reactions, Structures and Transport (FIRST) Center, an Energy Frontier Research Center funded by the U.S. Department of Energy, Office of 
Science, and Office of Basic Energy Sciences. We thank Pardha Saradhi Maram for some XRD and FTIR experiments.

\section{References}

[1] Mochalin VN, Shenderova O, Ho D, Gogotsi Y. The Properties and Applications of Nanodiamonds. Nature Nanotechnol. 2012; (7):11-23.

[2] Zou A, Wang MZ, Li YG, Zou LH, Yu H, Zhao YC. Analysis of structures and surface states of the nanodiamond particle synthesized by detonation. Micro \& Nano Letters 2009; 4(3):133-141.

[3] Wirth R, Rocholl A. Nanocrystalline diamond from Earth's mantle underneath Hawaii. Earth Planet Sc Lett 2003; 211:357-369.

[4] Nekhaev AI, Bagrii EI, Maximov AL. Petroleum nanodiamonds: new in diamondoid naphthenes. Petrol Chem+ 2010; 51(2):86-95.

[5] Lewis RS, Ming T, Wacker JE, Anders E, Steel E. Interstellar diamonds in meteorites. Nature 1987; 326:160-162.

[6] Allamandola LJ, Sandford SA, Tielens AGGM. Infrared spectroscopy of dense clouds in the C-H stretch region: methanol and "diamonds". Astrophys J 1992; 399, 134-146.

[7] Manuella FC. Can nanodiamonds grow in serpentinites-hosted hydrothermal systems? A theoretical modelling study. Mineral Maga 2013; 77(8), 3163-3174.

[8] Gogotsi YG, Nickel KG, Bahloul-Hourlier D, Merle-Mejean T, Khomenko GE, Skjerlie KP. Structure of carbon produced by hydrothermal treatment of $\beta$-SiC powder. J Mater Chem 1996; 6(4), 595-604.

[9] Gamarnik MY. Energetical preference of diamond nanoparticles. Phys Rev B 1996; 54(3), 2150-2156.

[10] Barnard AS, Russo SP, Snook IK. Size dependent phase stability of carbon nanoparticles: Nanodiamond versus fullerenes. J Chem Phys 2003; 118, 5094.

[11] Mote VD, Purushotham Y, Dole BN. Willimson-Hall analysis in estimation of lattice strain in nanometer-sized ZnO particles. J Theor Appl Phys 2012; 6(6):1-8.

[12] Schmidlin L, Pichot V, Comet M, Josset S, Rabu P, Spitzer D. Identification, quantification and modification of detonation nanodiamond functional groups. Diam Relat Mater 2012; 22:113117. 
[13] Levchenko AA, Kolesnikov AI, Trofymluk O, Navrotsky A. Energetics of single-wall carbon nanotubes as revealed by calorimetry and neutron scattering. Carbon 2011; 49(3):949-954.

[14] Costa GCC, McDonough JK, Gogotsi Y, Navrotsky A. Thermochemistry of onion-like carbons. Carbon 2014; 69:490-494.

[15] Costa GCC, Maram PS, Navrotsky A. Thermodynamics of nanoscale lead titanate and barium titanate perovskites. J Am Ceram Soc 2012; 95(10):3254-3262.

[16] Ji S, Jiang T, Xu K, Li S. FTIR study of the adsorption of water on ultradisperse diamond powder surface. Appl Surf Sci 1998; 133(4):231-238.

[17] Jiang T, Xu K. FTIR study of ultradispersed diamond powder synthesized by explosive detonation. Carbon 1995; 33(12):1663-71.

[18] Mochalin V, Osswald S, Gogotsi Y. Contribution of functional groups to the Raman spectrum of nanodiamond powders. Chem Mater 2009; 21(2): 273-279.

[19] Kolesov VP, Pimenova SM, Pavlovich VK, Tamm NB. Enthalpies of combustion and formation of fullerene C-60. J Chem Thermodyn 1996; 28(10):1121-5.

[20] Cherkasov NB, Savilov SV, Ivanov AS, Lunin VV. Bomb calorimetry as a bulk characterization tool for carbon nanostructures. Carbon 2013; 63:324-329.

[21] Chase MN. NIST-JANAF Thermochemical tables. J Phys Chem Ref Data 1985;14(1).

[22] Bale CW, Chartrand P, Degterov SA, Eriksson G, Hack K, Mahfoud RB, et al. FactSage thermochemical software and databases. Calphad 2002; 26(2):189-228.

[23] Chekhovskoi DV, Ivanisov AV, Proskurnev AY. A thermodynamic database and software system for the personal computer. IVTANTHERMO 1993.

[24] Robie RA, Hemingway BS. Thermodynamic properties of minerals and related substances at $298.15 \mathrm{~K}$ and 1 bar (10(5) pascals) pressure and at higher temperatures. U. S. Geological Survey Bulletin 1995; 2131.

[25] Costa GCC, Ushakov SV, Castro RHR, Navrotsky A, Muccillo R. Calorimetric Measurement of Surface and Interface Enthalpies of Yttria-Stabilized Zirconia (YSZ). Chem. Mater. 2010; 22(9):2937-2945. 
Table 1. Specific surface areas of nanodiamond measured by nitrogen adsorption (BET method) and crystallite sizes calculated from the specific surface area and from diffraction peak broadening.

\begin{tabular}{|c|c|c|c|c|c|}
\hline \multirow{2}{*}{\multicolumn{2}{|c|}{ Allotrope }} & \multirow{2}{*}{$\begin{array}{c}\text { BET SSA } \\
\left(\mathbf{m}^{2} \mathbf{g}^{-1}\right)\end{array}$} & \multirow{2}{*}{$\begin{array}{c}\text { BET Cryst. } \\
\operatorname{size}^{\mathrm{a}}(\mathbf{n m})\end{array}$} & \multicolumn{2}{|c|}{ XRD (Williamson - Hall) } \\
\hline & & & & $\begin{array}{c}\text { Crystallite }^{\mathbf{b}} \\
\text { size, }, \mathbf{n m}\end{array}$ & $\begin{array}{l}\text { Lattice } \\
\text { strain }^{\text {b }}\end{array}$ \\
\hline \multirow{4}{*}{$\begin{array}{l}\text { Carboxylated } \\
\text { Nanodiamonds }\end{array}$} & $\# 1$ & $314.52 \pm 0.74$ & $2.71 \pm 0.01$ & $4.5 \pm 0.4$ & 0.327 \\
\hline & $\# 2$ & $269.52 \pm 0.82$ & $3.17 \pm 0.01$ & $4.8 \pm 0.4$ & 0.020 \\
\hline & \#3 & $140.22 \pm 0.44$ & $6.09 \pm 0.02$ & $44.9 \pm 3.6$ & 0.401 \\
\hline & \#4 & $57.72 \pm 0.27$ & $14.78 \pm 0.08$ & $80.3 \pm 5.5$ & 0.151 \\
\hline
\end{tabular}

${ }^{\mathrm{a}}$ Calculated from BET SSA using theoretical densities $3.516 \mathrm{~g} / \mathrm{cm}^{3}$ (nanodiamond ICSD 650537). ${ }^{b}$ From X-ray diffraction pattern, crystallite size and lattice strain were calculated using Williamson-Hall plot. Extra digit is retained to prevent round-off error.

Table 2. Mass, carboxyl content (moles of carboxyl) and number of functional groups $/ \mathrm{nm}^{2}$ of the carboxylated nanodiamond samples.

\begin{tabular}{cccc}
\hline$\dagger$ Sample & Mass $(\mathbf{g})$ & $\begin{array}{c}{ }^{\mathbf{a}} \text { Carboxyl content } \\
(\mathbf{m o l})\end{array}$ & $\left.\begin{array}{c}{ }^{\mathbf{b}} \mathbf{C a r b o x y l}\left(\mathbf{C O O H} / \mathbf{n m}^{\mathbf{2}}\right) \\
\hline \# 1\end{array} 0\right)$ \\
$\# 2$ & $0.2000(2)$ & $0.000150(1)$ & $1.4(1)$ \\
$\# 3$ & $0.3850(2)$ & $0.000062(1)$ & $0.53(9)$ \\
$\# 4$ & $0.4039(2)$ & $0.000040(1)$ & $0.45(11)$ \\
\hline
\end{tabular}

$\dagger$ Uncertainties are given in parentheses and were propagated from the volumetric measurements

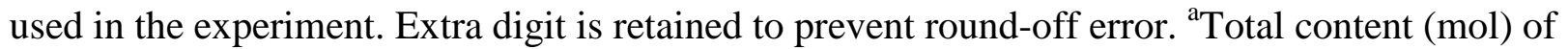
carboxyl of each sample determined by titration. ${ }^{\text {b }} \mathrm{COOH} / \mathrm{nm}^{2}=$ total content (mol)/Avogrado's number.total surface area. Total surface area $=$ sample mass.BET surface area (Table1). 
Table 3. Enthalpies of reaction ( $\left.\Delta H_{\text {reaction }}\right)$ and enthalpies relative to graphite plus $\mathrm{O}_{2}$ and $\mathrm{H}_{2}$ $\left(\Delta \mathrm{H}_{\mathrm{t}}\right)$ of carbon allotropes.

\begin{tabular}{|c|c|c|c|c|c|c|c|}
\hline \multicolumn{2}{|c|}{ Allotrope } & $\begin{array}{l}\dagger \Delta \mathbf{H}_{\text {reaction }} \\
-329.25(7)\end{array}$ & $\begin{array}{c} \pm \\
0.67\end{array}$ & $\frac{\dagger \Delta \mathbf{H}_{\mathbf{~ o x}, 25^{\circ} \mathrm{C}}}{-344.08}$ & $\frac{ \pm}{2.03}$ & $\frac{\dagger \Delta \mathbf{H}_{\mathbf{t}, 25^{\circ} \mathrm{C}}}{-50.4}$ & $\begin{array}{c} \pm \\
11.4\end{array}$ \\
\hline $\begin{array}{l}\text { Carboxylated } \\
\text { Nanodiamond }\end{array}$ & $\begin{array}{l}\# 1 \\
\# 2 \\
\# 3 \\
\# 4\end{array}$ & $\begin{array}{l}-329.25(7) \\
-328.03(5) \\
-358.90(9) \\
-365.97(7)\end{array}$ & $\begin{array}{l}0.67 \\
1.16 \\
1.13 \\
0.84\end{array}$ & $\begin{array}{l}-344.08 \\
-341.78 \\
-372.55 \\
-379.45\end{array}$ & $\begin{array}{l}2.03 \\
2.19 \\
1.99 \\
1.82\end{array}$ & $\begin{array}{l}-50.4 \\
-52.1 \\
-21.1 \\
-14.1\end{array}$ & $\begin{array}{l}11.4 \\
10.5 \\
7.2 \\
6.8\end{array}$ \\
\hline${ }^{\mathrm{a}}$ Carbon Onion & $\begin{array}{l}\text { UD90, } 1800^{\circ} \mathrm{C} \\
\text { UD50, } 1800^{\circ} \mathrm{C} \\
\text { UD50, } 1500^{\circ} \mathrm{C} \\
\text { UD50, } 1300^{\circ} \mathrm{C}\end{array}$ & $\begin{array}{l}-396.04(6) \\
-397.29(6) \\
-393.87(5) \\
-382.67(5)\end{array}$ & $\begin{array}{l}0.67 \\
1.07 \\
0.54 \\
0.78\end{array}$ & $\begin{array}{l}-408.14 \\
-409.39 \\
-405.97 \\
-394.77\end{array}$ & $\begin{array}{l}0.68 \\
1.07 \\
0.54 \\
0.78\end{array}$ & $\begin{array}{c}14.62 \\
15.87 \\
12.44 \\
1.25\end{array}$ & $\begin{array}{l}0.69 \\
1.08 \\
0.54 \\
0.78\end{array}$ \\
\hline${ }^{\mathrm{b}}$ Fullerene, $\mathrm{C}_{60}$ & & - & - & -432.76 & 0.19 & 39.23 & 0.20 \\
\hline 'Single-wall carb & nanotubes & - & - & - & - & 7.00 & 1.00 \\
\hline $\begin{array}{l}{ }^{\mathrm{d}} \text { Cylindrical mul } \\
\text { nanotubes }\end{array}$ & alled carbon & - & - & - & - & 8.60 & 0.52 \\
\hline $\begin{array}{l}{ }^{\mathrm{d}} \text { Herringbone mı } \\
\text { nanotubes }\end{array}$ & walled carbon & - & - & - & - & 21.70 & 1.32 \\
\hline${ }^{\mathrm{d}}$ Carbon fibers & & - & - & - & - & 16.56 & 2.76 \\
\hline Graphite $^{\mathrm{e}}$ & & - & - & -393.52 & 0.05 & 0 & - \\
\hline Diamond $^{\mathrm{f}}$ & & - & - & -395.42 & 0.50 & 1.89 & 0.05 \\
\hline
\end{tabular}

$\dagger \Delta \mathrm{H}_{\text {reaction }}$ is the measured enthalpy when a sample is dropped from room temperature into the calorimeter at $800{ }^{\circ} \mathrm{C}$ and oxidized to $\mathrm{CO}_{2}$. $†$ Mean values of the number of experiments are given in parentheses, uncertainties are calculated as two standard deviations of the mean, and extra digit is retained to prevent round-off error. ${ }^{\mathrm{a}}[14],{ }^{\mathrm{b}}[19],{ }^{\mathrm{c}}[13],{ }^{\mathrm{d}}[20],{ }^{\mathrm{e}}[21]$ and ${ }^{\mathrm{f}}[22]$. Note that enthalpy of formation at $25^{\circ} \mathrm{C}$ is the same as enthalpy of transition from graphite at $25^{\circ} \mathrm{C}$ to the carbon allotrope of interest. 
Table 4. Thermodynamic cycle used to calculate the enthalpies of oxidation of the samples at $25^{\circ} \mathrm{C}$.

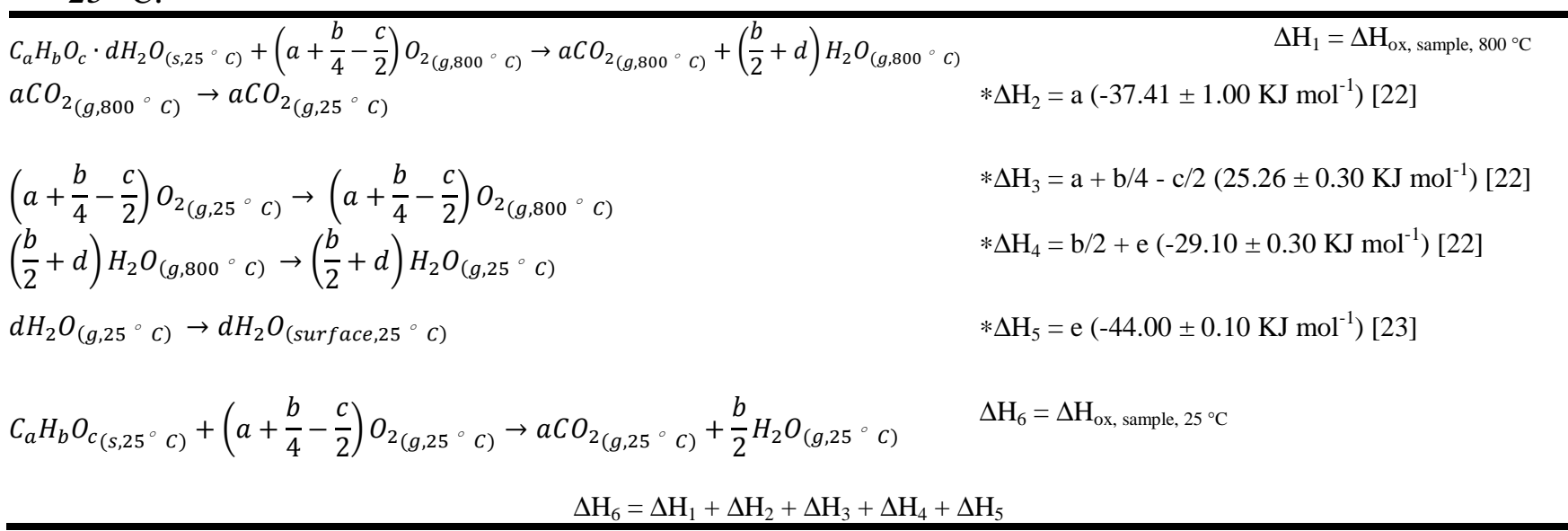

*Extra digit is retained to prevent round-off error.

Table 5. Content of carbon, oxygen, hydrogen and water in the carboxylated nanodiamond samples.

\begin{tabular}{ccccc}
\hline \multirow{十}{*}{ Sample } & ${ }^{\mathrm{a}} \mathbf{C}$ & ${ }^{a} \boldsymbol{H}$ & ${ }^{a} \boldsymbol{O}$ & ${ }^{b} \boldsymbol{H}_{2} \boldsymbol{O}(\mathbf{m o l})$ \\
& $\boldsymbol{a}$ & $\boldsymbol{b}$ & $\boldsymbol{c}$ & $\boldsymbol{d}$ \\
\hline$\# 1$ & $1.000(29)$ & $0.00765(59)$ & $0.0153(12)$ & $0.03318(96)$ \\
$\# 2$ & $1.000(27)$ & $0.00297(49)$ & $0.00594(98)$ & $0.02057(55)$ \\
$\# 3$ & $1.000(18)$ & $0.00132(33)$ & $0.00263(66)$ & $0.01987(37)$ \\
$\# 4$ & $1.000(17)$ & $0.00058(31)$ & $0.00116(63)$ & $0.01804(32)$ \\
\hline
\end{tabular}

$\dagger$ Uncertainties are given in parentheses, and extra digit is retained to prevent round-off error. ${ }^{a}$ There are no units for $a, b$ and $c$ since they are numbers in a chemical formula although they were calculated from the molar fraction of their elements. $b$ and $c$ are calculated assuming the total amount of carboxyl groups (Table 2) and are normalized for $1 \mathrm{~mol}$ of carbon. Water was determined by gravimetric analysis and was also normalized for 1 mol of carbon.

Table 6. Thermodynamic cycle used to calculate the enthalpies of formation of the samples at $25^{\circ} \mathrm{C}$.

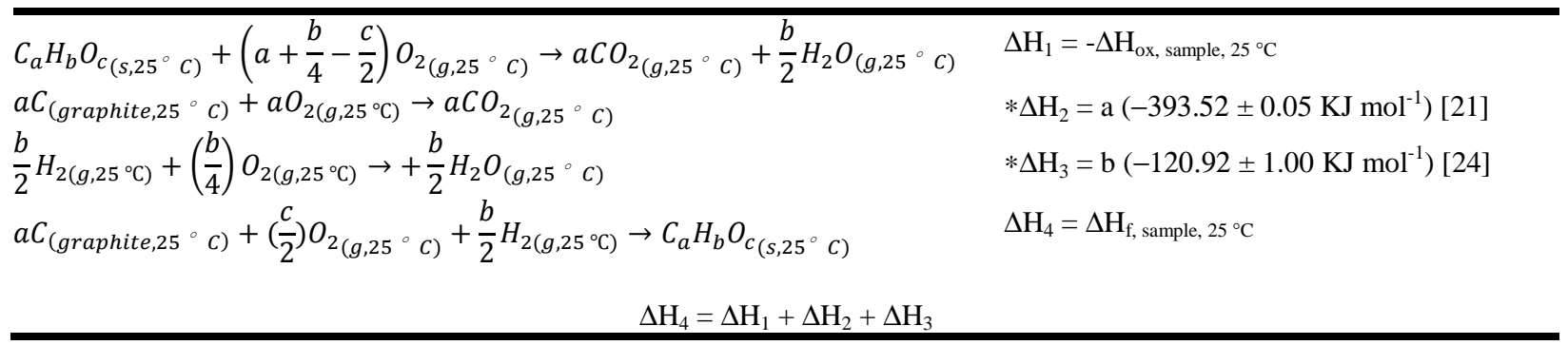

*Extra digit is retained to prevent round-off error. 


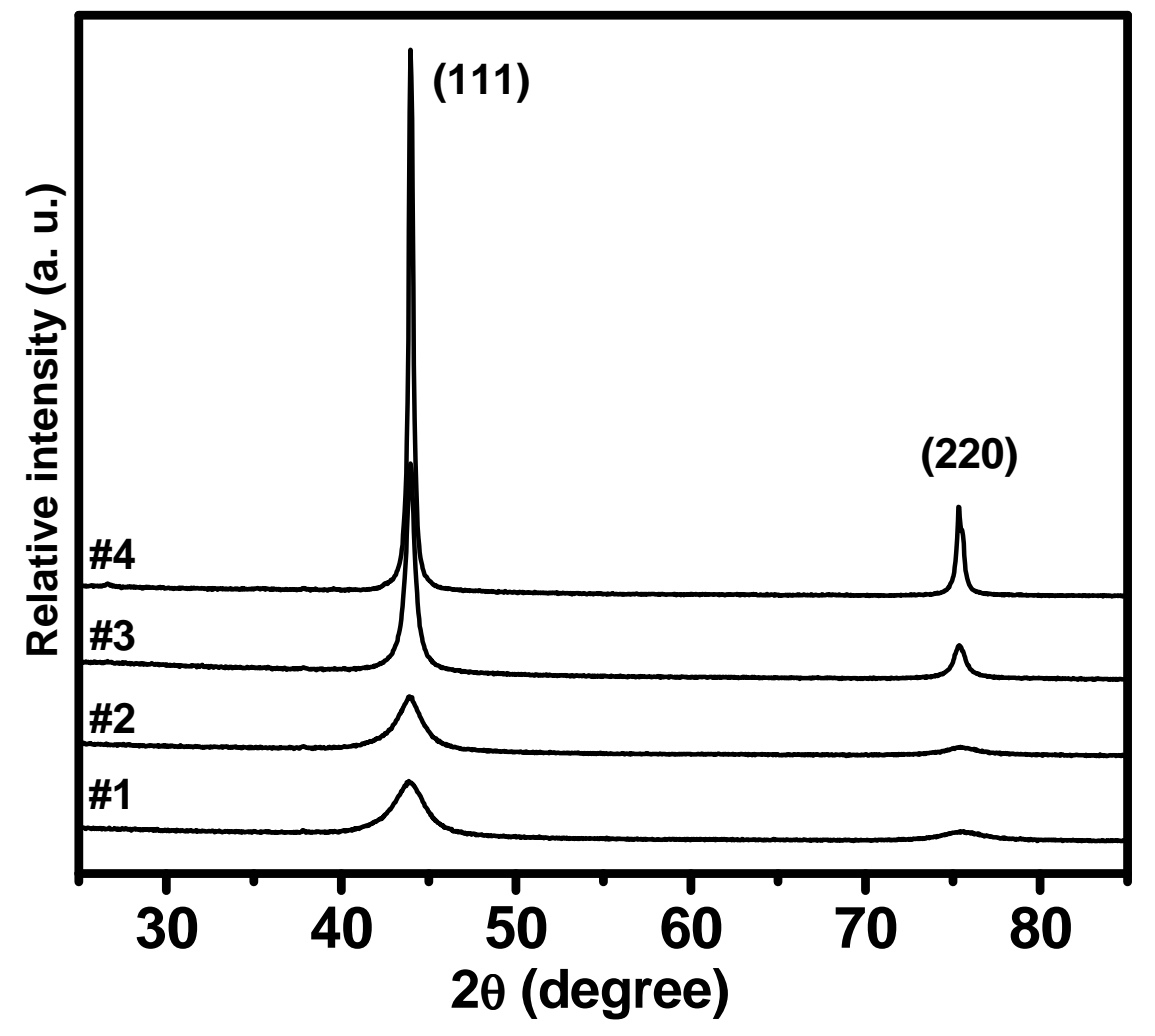

Figure 1. XRD patterns of the carboxylated nanodiamond powder samples used for high temperature oxidation calorimetry. Crystallite sizes of the samples are given in Table 1. 


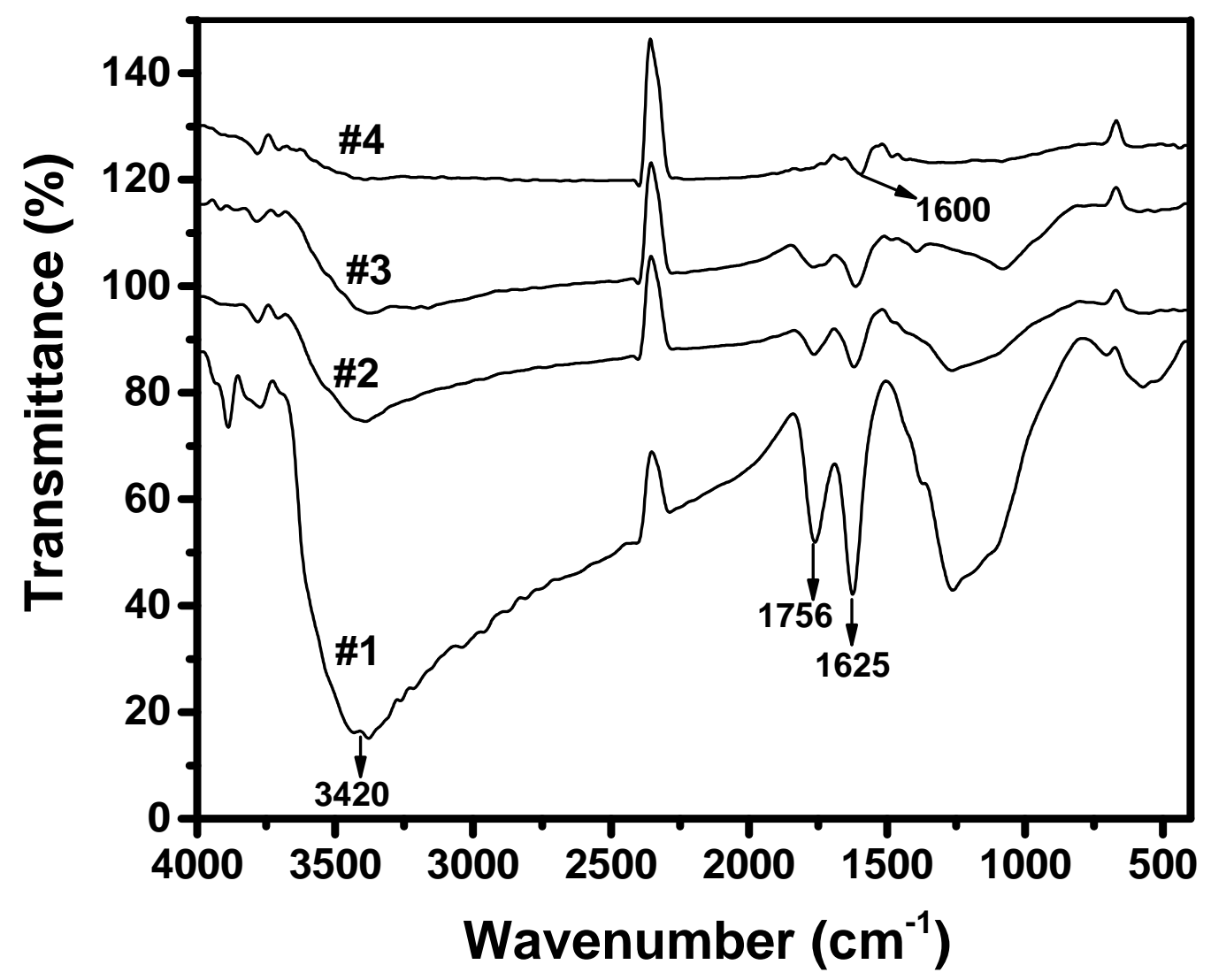

Figure 2. FTIR spectra of the carboxylated nanodiamond samples. Negative transmittance is observed in some spectra due to traces of water and carbon dioxide present in the background. 


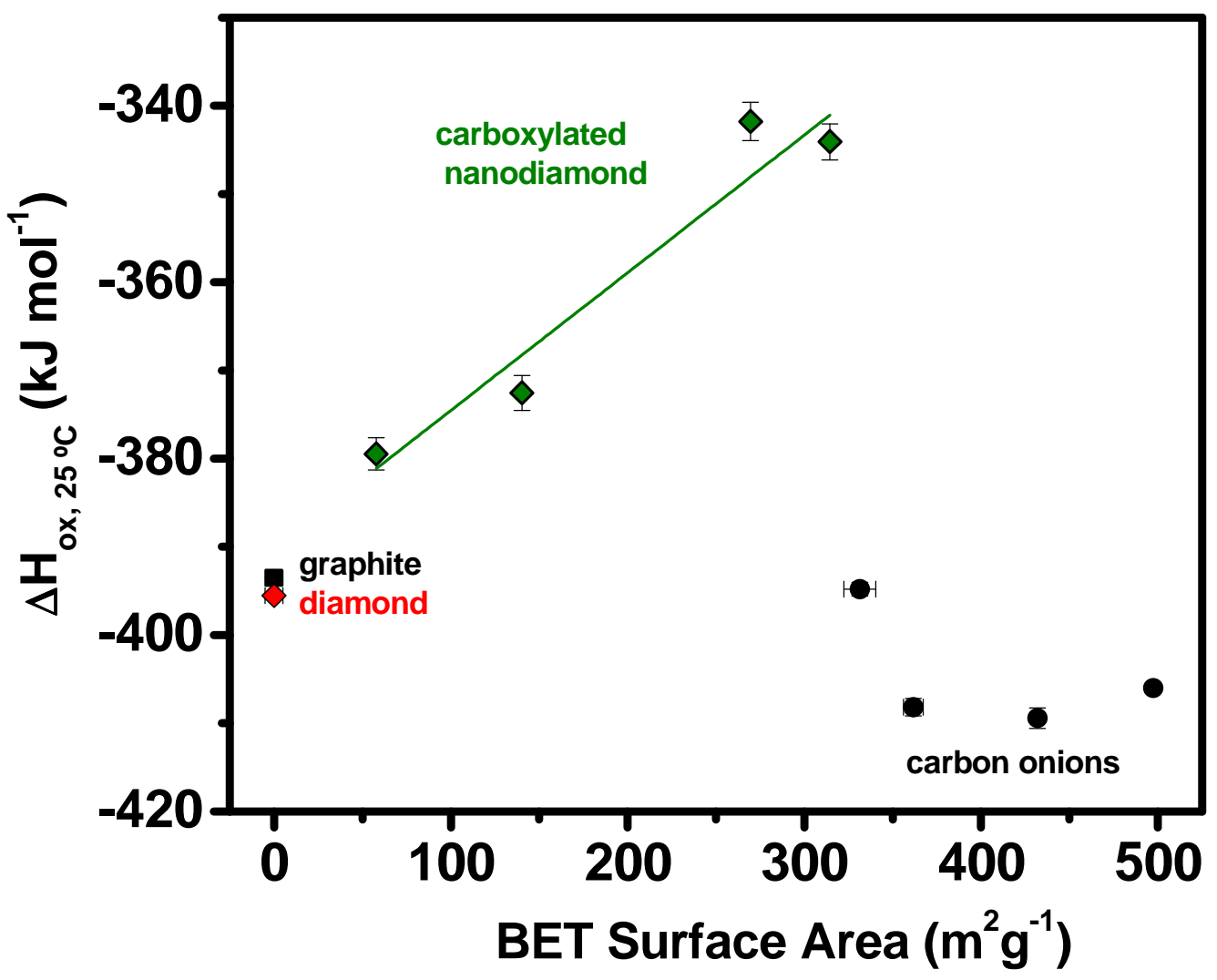

Figure 3. Enthalpies of oxidation of the carbon allotropes versus their BET surface area. Trace line is added to act as guide to the eyes. 


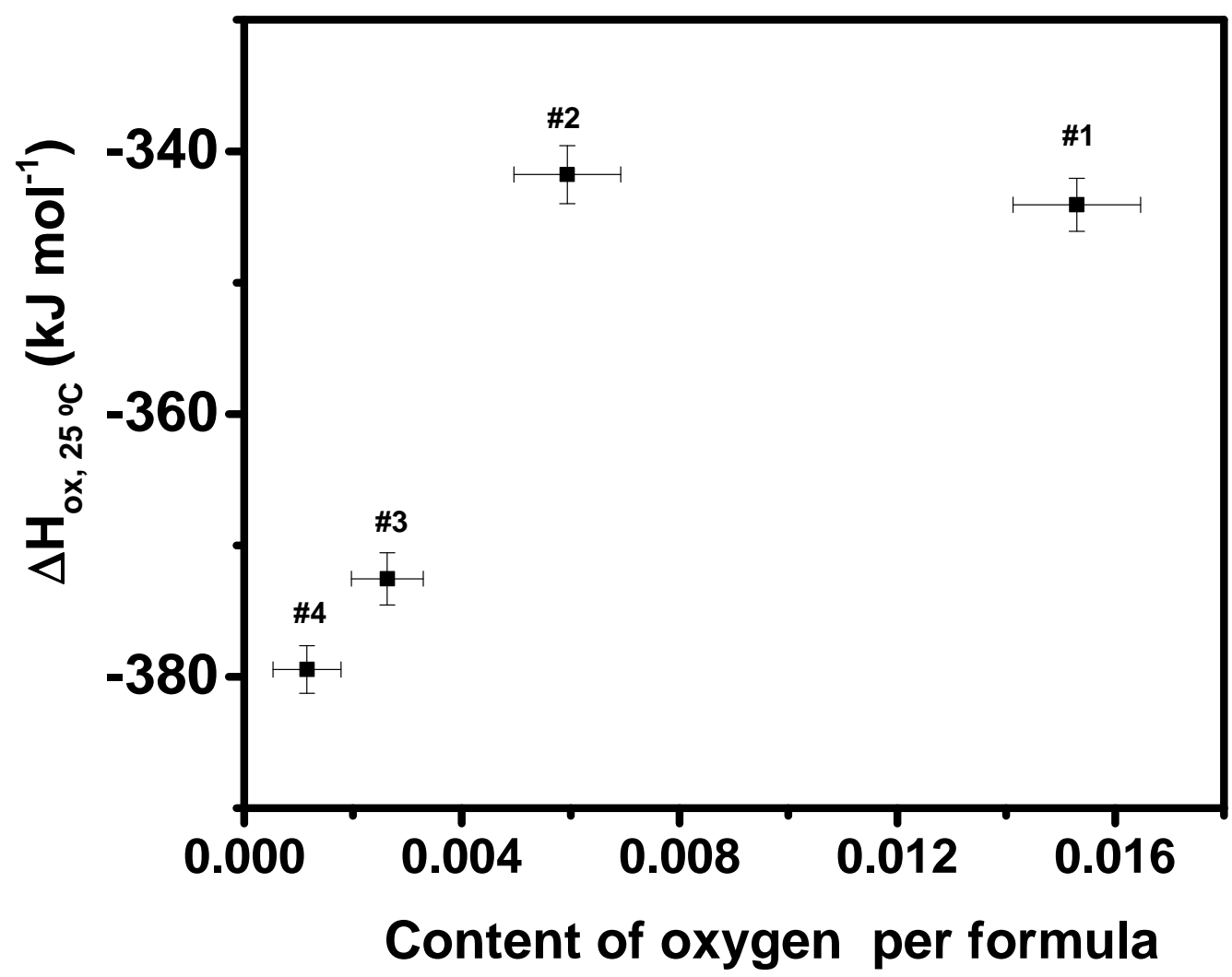

Figure 4. Enthalpies of oxidation of the nanodiamond samples versus the content of oxygen in $\mathrm{C}_{\mathrm{a}} \mathrm{H}_{\mathrm{b}} \mathrm{O}_{\mathrm{c}}$ (c in Table 5). 